\title{
Multimodality treatment of stage II thymic tumours
}

\author{
Carolina Carillo $^{1}$, Daniele Diso ${ }^{1}$, Sara Mantovani ${ }^{1}$, Ylenia Pecoraro ${ }^{1}$, Tiziano De Giacomo ${ }^{1}$, Anna \\ Maria Ciccone ${ }^{2}$, Camilla Poggi ${ }^{2}$, Flavia Longo ${ }^{3}$, Raffaele Cassese ${ }^{4}$, Vincenzo Tombolini ${ }^{4}$, Erino Angelo \\ Rendina $^{2}$, Federico Venuta ${ }^{1}$, Marco Anile ${ }^{1}$
}

${ }^{1}$ Department of Thoracic Surgery, University of Rome Sapienza, Rome, Italy; ${ }^{2}$ Department of Thoracic Surgery, Sant'Andrea Hospital, Rome, Italy; ${ }^{3}$ Department of Medical Oncology, ${ }^{4}$ Department of Radiotherapy, Policlinico Umberto I, "Sapienza” University of Rome, Rome, Italy

Contributions: (I) Conception and design: F Venuta; (II) Administrative support: None; (III) Provision of study materials or patients: None; (IV) Collection and assembly of data: All authors; (V) Data analysis and interpretation: None; (VI) Manuscript writing: All authors; (VII) Final approval of manuscript: All authors.

Correspondence to: Federico Venuta, MD. Cattedra di Chirurgia Toracica, Policlinico Umberto I, Università di Roma Sapienza, V.le del Policlinico 155, 00161 Roma, Italy. Email: federico.venuta@uniroma1.it.

Background: Complete resection for stage II thymic tumors can be easily accomplished even if the capsula
and adjacent mediastinal tissue are macroscopically involved; however, also at this stage, recurrence may
occur, particularly for B2, B3 and thymic carcinoma. The criteria for the administration of adjuvant therapy
remain controversial and it is unclear whether patients at this stage may benefit from it. We reviewed a series
of patients at this stage receiving adjuvant chemo-radiotherapy (chemo-RT) based on histology.

Methods: Eighty-eight consecutive patients with stage II thymic tumors were reviewed; 59 patients $(67 \%)$ with B thymoma or thymic carcinoma received adjuvant treatment with mediastinal irradiation (40-55 Gy), chemotherapy $(\mathrm{CH})$ (PAC regimen) or a combination of both.

Results: Complete resection was achieved in all patients. Fifty-four patients (61\%) received post-operative chemo-RT, 2 (2\%) patients received adjuvant $\mathrm{CH}$ only and 3 (3\%) post-operative RT only; they all had B2, B3 histology or thymic carcinoma. The median follow up was $107 \pm 83$ months. 5-year and 10-year survival were $96 \% \pm 2 \%$ and $83.4 \% \pm 5 \%$. Recurrence was observed in 5 patients $(5.7 \%)$. Disease-free 5 and 10 -year survival was $94 \% \pm 2 \%$ and $92 \% \pm 3 \%$ respectively. Five patients $(5.7 \%)$ had recurrence.

Conclusions: The administration of adjuvant chemo-RT to patients with stage II type B thymoma and thymic carcinoma contributes to reduce the recurrence rate and to increase long-term survival.

Keywords: Thymoma; thymic carcinoma; adjuvant therapy; radiotherapy (RT); chemotherapy (CH); Masaoka stage II

Submitted Nov 24, 2016. Accepted for publication Jun 21, 2017.

doi: $10.21037 /$ jtd.2017.06.116

View this article at: http://dx.doi.org/10.21037/jtd.2017.06.116

\section{Introduction}

Thymic tumors are relatively rare neoplasms arising from the epithelial thymic cells. They show a significant histological heterogeneity and clinical behavior ranging from an indolent non-invasive attitude to a highly infiltrative and metastasizing one. Prognosis is affected by the Masaoka-Koga staging, the World Health Organization (WHO) histological classification, completeness of resection, the diameter of the lesion and the presence of vascular invasion (1-8).

Complete surgical resection, remains the gold standard for cure at any stage. Chemotherapy $(\mathrm{CH})$ is usually administered as an induction before surgery or as definitive treatment when the tumor is deemed unresectable. Radiotherapy (RT) is indicated in selected patients, particularly as adjuvant treatment. A multimodality approach is beneficial for a selected group of patients with invasive tumors (9-11).

According to the Masaoka-Koga staging system, 
Table 1 Masaoka stage $v s$. World Health Organization histology

\begin{tabular}{lccc}
\hline \multirow{2}{*}{ WHO histology } & \multicolumn{2}{c}{ Masaoka stage } & \multirow{2}{*}{ Recurrence } \\
\cline { 2 - 3 } & Stage IIA (\%) & Stage IIB (\%) & \\
\hline A & $7(20.0)$ & $4(7.6)$ & - \\
AB & $8(22.9)$ & $7(13.2)$ & - \\
B1 & $1(2.9)$ & $2(3.8)$ & - \\
B2 & $10(28.6)$ & $12(22.6)$ & $2(9.1)$ \\
B3 & $7(20.0)$ & $23(43.4)$ & $2(6.7)$ \\
Thymic carcinoma & $2(5.6)$ & $5(9.4)$ & $1(14.3)$ \\
Total & $35(39.8)$ & $53(60.2)$ & $5(5.7)$ \\
\hline
\end{tabular}

thymic tumors are classified as stage IIA when they show microscopic trans-capsular invasion and IIB in case of macroscopic invasion of the surrounding mediastinal fat tissue or gross adherence to the mediastinal pleura $(12,13)$. Although complete resection can be easily achieved at this stage, local recurrence and metastatic spread may be observed, particularly in case of B2, B3 and thymic carcinoma $(6,7,14,15)$. For this reason, some authors suggest the administration of adjuvant RT to improve local control (16-20). However, the clinical benefit of adjuvant $\mathrm{RT}$ in this setting still remains controversial; in fact, not only local recurrence, but also distant metastatic spread may occur. We have previously described our initial experience with adjuvant CH-RT for stage III tumors to prevent local recurrence and distant spreading (9,21-25). Up to 1988 we have treated stage II thymic tumors with surgical resection only; post-operative RT was rarely administered and without standardized indications. We observed a relatively high number of recurrences (33\%) and most of them were outside the mediastinum $(21,23)$; for this reason, since 1989 we administer adjuvant CH-RT for stage II-WHO type B thymoma and thymic carcinoma. The aim of this study is to assess the potential benefit of this approach.

\section{Methods}

Eighty-eight patients prospectively entered the database between 1989 and 2013. The study was approved by the ethical committee of our institution (prot. 116/10). All tumors were staged according to the Masaoka-Koga system. Histology was assessed according to the WHO histological classification (25).

Forty-seven patients were male $(53.4 \%)$; the mean age was $57 \pm 16$ years (range, $22-87$ years) and $32(36 \%)$ were affected by Myasthenia Gravis; one patient had pemphigus and one had hypogammaglobulinemia. Eighteen patients $(20.4 \%)$ referred tumor-related symptoms as chest pain and tightness; 22 patients $(25 \%)$ presented comorbidities including arrhythmias (6\%), diabetes $(9 \%)$ and chronic obstructive pulmonary disease $(10 \%)$. Three patients (3.4\%) had a previous history of cancer (breast, prostate and leukemia). Histology and stage are reported in Table 1. The median follow-up was $107 \pm 83$ months, ranging from 8 to 280 months. No patient was lost to follow-up.

According to our protocol, patients with stage IIA tumors and B2, B3 or C histology received adjuvant $\mathrm{CH}-\mathrm{RT}$; in case of B1 thymoma adjuvant therapy was administered only for lesions with a diameter $>5 \mathrm{~cm}$. In case of stage IIB, all patients with B or C histology underwent adjuvant treatment. $\mathrm{CH}$ was administered with three cycles of PAC (cisplatinum $50 \mathrm{mg} / \mathrm{m}^{2}$, doxorubicin $50 \mathrm{mg} / \mathrm{m}^{2}$, and cyclophosphamide $500 \mathrm{mg} / \mathrm{m}^{2}$ ); mediastinal RT was administered at a dose between 45 and 55 Gy.

Descriptive statistics were reported as means with ranges and standard deviation for continuous variables and as frequencies and proportions for categorical variables. Disease-free survival (DFS) was defined as the time from surgery to recurrence. Overall survival (OS) was defined as the time from surgery to death by any cause. The KaplanMeier method was used to estimate DFS and OS. Results were considered significant for a $\mathrm{P}$ value less than 0.05 . The statistical analysis was performed with SPSS Statistics 17.0 (IBM ${ }^{\circledR}$-ITALIA).

\section{Results}

Complete resection was achieved in all patients and no perioperative mortality was observed. Surgical data are reported in Table 2. Twenty-six patients (29.5\%) had type $\mathrm{A}$ or $\mathrm{AB}$ thymoma and they did not receive any adjuvant treatment. Fifty-four patients $(61 \%)$ received post-operative $\mathrm{CH}-\mathrm{RT}$, including one B1/IIA patient (tumor diameter $>5 \mathrm{~cm}$ ) (Table 2); 2 (2\%) received adjuvant $\mathrm{CH}$ only and 3 (3\%) postoperative RT only. Two patients with B2 histology refused any adjuvant treatment. One patient with B3 histology did not receive any adjuvant treatment due to her advanced age ( 87 years).

Hematologic toxicity was observed in 5 patients with mild temporary leukopenia; non-hematologic toxicity included stomatitis, alopecia, nausea and vomiting. There were no infectious complications. Post RT esophagitis was 
Table 2 Treatment and complications of the patients $(\mathrm{n}=88)$

\begin{tabular}{ll}
\hline Variable & Number [\%] \\
\hline Induction chemotherapy & $2[2]$ \\
Surgery alone & $29[33]$ \\
Adjuvant treatment & \\
Adjuvant CH + RT & $54[61]$ \\
Adjuvant CH & $2[2]$ \\
Adjuvant RT & $3[3]$ \\
Type of surgery & \\
Sternotomy & $78[89]$ \\
Thoracotomy & $7[8]$ \\
VATS & $3[3]$ \\
Complications & $1[1]$ \\
Pneumothorax & $1[1]$ \\
Bleeding & $1[1]$ \\
Sternal dehiscence & \\
Myasthenia gravis crisis & $14]$ \\
Respiratory failure & \\
Deaths & $110]$ \\
Tumor related & \\
Other causes & \\
\hline CH, chemotherapy; RT, radiotherapy; VATS, video assisted
\end{tabular}

$\mathrm{CH}$, chemotherapy; $\mathrm{RT}$, radiotherapy; VATS, video assisted thoracic surgery.

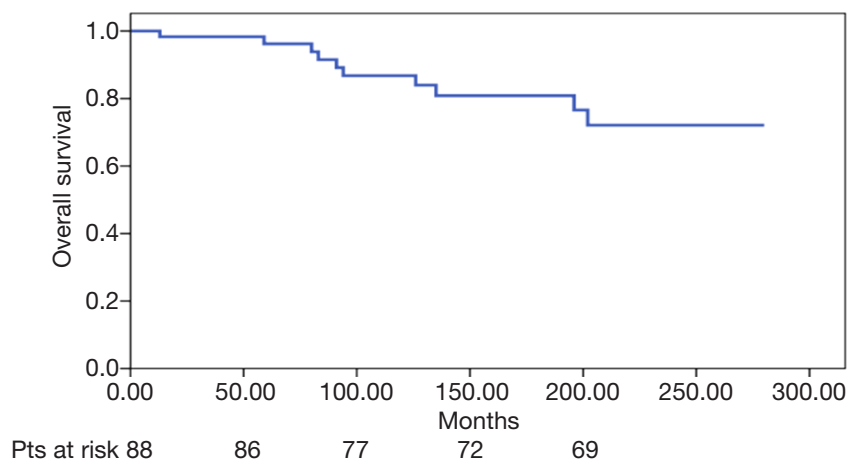

Figure 1 Overall survival of the study population.

observed in one patient.

OS and DFS are reported in Figures 1,2. Recurrence was observed in 5 patients (5.7\%) $105 \pm 83$ months (range, 8-280 months) after surgery (Table 1). One patient had

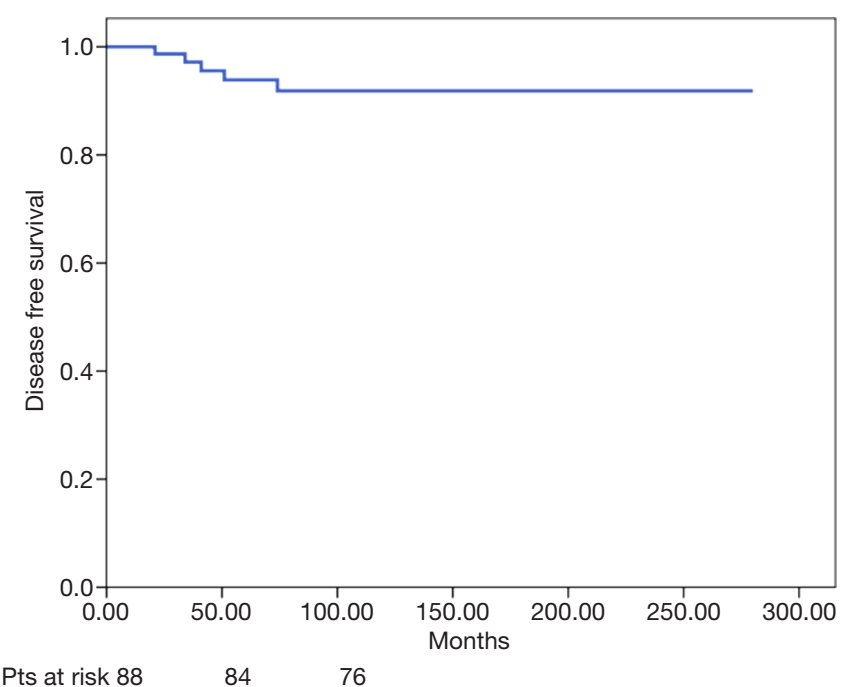

Figure 2 Disease free survival of the study population.

mediastinal recurrence and he was treated with $\mathrm{CH}$; two patients had extrathoracic recurrence (retroperitoneal and liver); two showed intrathoracic recurrence (pleural after thoracoscopic thymectomy and lung), both of them underwent re-resection.

During follow-up 13 patients died (15\%): 4 of them (4.5\%) for disease progression; the others died for nonthymoma related complications [one cardiac failure, two acute myocardial infarction (AMI), one herpetic encephalitis, one metastatic melanoma, four "senectus"]. We did not observe any statistically significant difference in terms of OS between stage IIA and IIB after $1989(\mathrm{P}=0.1)$.

\section{Discussion}

The Masaoka-Koga staging system is based on the detection of microscopic or macroscopic invasion into the capsula of the tumor and adjacent structures and on the presence of metastatic disease. Although for stage II tumors complete resection is always feasible, incomplete resection has been reported and local or distant recurrence may occur. Furthermore, the literature supports the assumption that thymic tumors show a morphologic continuum: some of them may progress from $\mathrm{B} 1$ to $\mathrm{B} 3$ and even to thymic carcinoma $(5,22)$. These data are sustained also by the finding of different histologies in the same specimen.

In a previous study, in a population of 27 stage IIB patients we observed a relatively high recurrence rate (33\%) in the subgroup IIB (21); most of those recurrences 


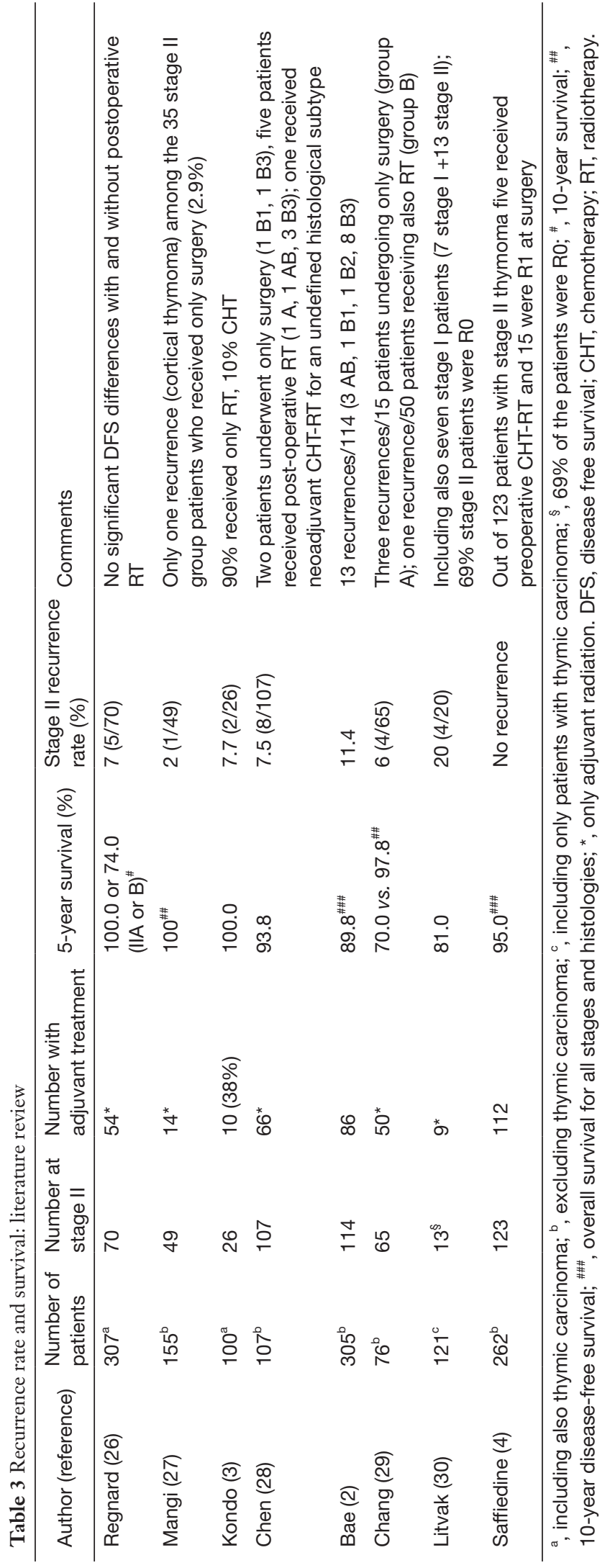

were outside the mediastinum (pleura, lung, liver, bone), confirming that even at this stage thymoma can recur and metastasize. For this reason, at that time we postulated that also stage II thymic tumors might benefit from adjuvant treatment. Thus, RT and $\mathrm{CH}$ were administered with the aim of reducing local and distant recurrence respectively. The results of our study show that the recurrence rate is decreased and DFS seems improved after adjuvant treatment, when compared to our previous data (23) and the international literature (also when postoperative RT only is administered) (Table 3).

Bae showed a recurrence rate of $11.4 \%$ (13 out of 114 patients) (2); Detterbeck reported an average recurrence rate of $11 \%$ for stage II (31). Both studies included stage II patients and only RT was administered after surgery. Other studies are much more difficult to interpret since a thorough analysis is not always reported: in other words, it is not always clear how many tumors are at stage IIA or IIB as well as the distribution of histology, particularly the incidence of thymic carcinoma; also the exact number of patients at this stage receiving adjuvant $\mathrm{RT}$ is difficult to extrapolate. Furthermore, recurrence is not always well reported at this stage. In the present study the overall recurrence rate was $5.7 \%$ while it was $33 \%$ in our previous series with surgery alone. Recurrence was observed only in type C (1), B3 (2) and B2 (2) (Table 1). This finding supports that adjuvant $\mathrm{CH}-\mathrm{RT}$ might contribute to reduce the recurrence rate also at this stage and particularly for more aggressive histological subtypes. It also confirms that the WHO classification has a prognostic impact on this variable: as a matter of fact recurrence occurs only in type B2-3 and thymic carcinoma patients. In the present series OS is influenced by other causes than tumor (cardiac ischemia or failure, encephalitis, second malignancies, older age) and only 4 patients (4.5\%) died for disease progression. The consistency of this data is supported by the long follow-up (107 \pm 83 months).

We have observed 9 (10.2\%) metachronous extra-thymic malignancies (thyroid, breast, liver, lung, skin, prostate and leukemia); they were associated with B2 and B3 histology. This finding supports the hypothesis that more aggressive histological subtypes are associated with a higher risk of developing other malignancies and poorer survival (32). The potential role of adjuvant treatment in the development of other malignancies deserves further investigation.

Thymic carcinoma is relatively rare $(15 \%$ to $20 \%$ of all thymic tumors) $(1,3)$ and it shows a spectrum of 11 subtypes (25). In most of the cases it behaves aggressively and it is discovered at an advanced stage, with a lower 
survival rate (30-60\% at 5 years; $17 \%$ if unresectable) (33). It is now considered as a separate entity. In our early stage series, we observed only 7 (8\%) patients with thymic carcinoma. Considering this group separately, only 1 (14.2\%) patient had recurrence; this incidence is low when compared with the current literature $(7,27,30)$ (Table 3). On the other side, excluding type C, the recurrence rate (4/81) was further reduced respect to our previous series not including this histological subtype (4.9\% vs. $33 \%)$. These results contribute to support a multidisciplinary approach also at this stage. Although the 2015 ESMO guidelines support the administration of postoperative RT only (34), we believe that also $\mathrm{CH}$ should be added in this specific subset of patients since in the literature, and also in our previous experience, distant metastases have been observed and reported.

In conclusion, we confirm that $\mathrm{A}$ and $\mathrm{AB}$ thymoma show an excellent prognosis with no recurrence, whereas $\mathrm{B}$ and $\mathrm{C}$ tumors are usually more aggressive also at early stage; postoperative CH-RT might contribute to improve prognosis. Nonetheless, this study does not allow definitive conclusions due to the limitations related to the small number of patients, particularly those with thymic carcinoma; however, with all the limitations of this report, it should serve as "food for thought" for future investigations.

\section{Acknowledgements}

None.

\section{Footnote}

Conflicts of Interest: The authors have no conflicts of interest to declare.

Ethical Statement: The study was approved by the Ethical Committee of the University of Rome Sapienza - Policlinico Umberto I (prot. 116/10). Written informed consent was obtained from the patient.

\section{References}

1. Venuta F, Rendina EA, Anile M, et al. Thymoma and thymic carcinoma. Gen Thorac Cardiovasc Surg 2012;60:1-12.

2. Bae MK, Lee CY, Lee JG, et al. Predictors of recurrence after Thymoma resection. Yonsei Med J 2013;54:875-82.

3. Ruffini E, Detterbeck F, Van Raemdonck D, et al. Thymic carcinoma: a cohort study of patients from the European Society of Thoracic Surgeons database. J Thorac Oncol 2014;9:541-8.

4. Safieddine N, Liu G, Cuningham K, et al. Prognostic factors for cure, recurrence and long-term survival after surgical resection of thymoma. J Thorac Oncol 2014;9:1018-22.

5. Ströbel P, Marx A, Zettl A, et al. Thymoma and thymic carcinoma: an update of the WHO Classification 2004. Surg Today 2005;35:805-11.

6. Okumura M, Ohta M, Tateyana H, et al. The World Health Organization histologic classification system reflects the oncologic behaviour of thymoma: a clinical study of 273 patients. Cancer 2002;94:624-32.

7. Kondo K, Yoshizawa K, Tsuyuguchi M, et al. WHO histologic classification is a prognostic indicator in thymoma. Ann Thorac Surg 2004;77:1183-8.

8. Guerrera F, Rendina EA, Venuta F, et al. Does the World Health Organization histological classification predict outcomes after thymomectomy? Results of a multicenter study on 750 patients. Eur J Cardiothorac Surg 2015;48:48-54.

9. Venuta F, Rendina EA, Longo F, et al. Long-term outcome after multimodality treatment for stage III thymic tumors. Ann Thorac Surg 2003;76: 1866-72.

10. Leuzzi G, Rocco G, Ruffini E, et al. Multimodality therapy for locally advanced thymomas: a propensity score-matched cohort study from the European Society of Thoracic Surgeons Database. J Thorac Cardiovasc Surg 2016;151:47-57.e1.

11. Venuta F, Anile M, Rendina EA, et al. The value of transcapsular invasion in patients with thymoma. Arch Pathol Lab Med 2009;133:1364-5.

12. Detterbeck FC, Nicholson AG, Kondo K, et al. The Masaoka-Koga stage classification for thymic malignancies: clarification and definition of terms. J Thorac Oncol 2011;6:S1710-6.

13. Nicholson AG, Detterbeck FC, Marino M, et al. The IALSC-ITMIG thymic epithelial tumors staging project: proposals for the $\mathrm{T}$ component for the forthcoming $(8 \mathrm{TH})$ edition of the TNM classification of malignant tumors. J Thorac Oncol 2014;9:S73-80.

14. Haniuda M, Kondo R, Numanami $\mathrm{H}$, et al. Recurrence of thymoma: clinicopathological features, re-operation and outcome. J Surg Oncol 2001;78:183-8.

15. Gripp S, Hilgers K, Wurm R, et al. Thymoma: prognostic factors and treatment outcomes. Cancer 1998;83:1495-503.

16. Berman AT, Litzky L, Livolsi V, et al. Adjuvant 
radiotherapy for completely resected stage II thymoma. Cancer 2011;117:3502-8.

17. Jackson MA, Ball DL. Post-operative radiotherapy in invasive thymoma. Radiother Oncol 1991;21:77-82.

18. Curran WJ Jr, Kornstein MJ, Brooks JJ, et al. Invasive thymoma: the role of mediastinal irradiation following complete and incomplete surgical resection. J Clin Oncol 1988;6:1722-27.

19. Ogawa K, Toita T, Kakinohana Y, et al. Postoperative radiation therapy for completely resected invasive thymoma: prognostic value of pleural invasion for intrathoracic control. Jpn J Clin Oncol 1999;29:474-8.

20. Nakahara K, Ohno K, Hashimoto J, et al. Thymoma: results with complete resection and adjuvant postoperative irradiation in 141 consecutive patients. J Thorac Cardiovasc Surg 1988;95:1041-7.

21. Pescarmona E, Rendina EA, Venuta F, et al. Analysis of prognostic factors and clinic-pathological staging of thymoma. Ann Thorac Surg 1990;50:534-8.

22. Pescarmona E, Rendina EA, Venuta F, et al. Recurrent thymoma: evidence for histological progression. Histopathology 1995;27:445-9.

23. Venuta F, Rendina EA, Pescarmona E, et al. Multimodality treatment of thymoma: a prospective study. Ann Thorac Surg 1997;64:1585-91.

24. Ruffini E, Van Raemdonck D, Detterbeck F, et al. Management of thymic tumors: a survey of current practice among members of the European Society of Thoracic Surgeons. J Thorac Oncol 2011;6:614-23.

25. Travis WD, Brambilla E, Muller-Hermelink HK, et al. WHO classification of tumors, pathology and genetics of

Cite this article as: Carillo C, Diso D, Mantovani S, Pecoraro Y, De Giacomo T, Ciccone AM, Poggi C, Longo F, Cassese R, Tombolini V, Rendina EA, Venuta F, Anile M. Multimodality treatment of stage II thymic tumours. J Thorac Dis 2017;9(8):2369-2374. doi: 10.21037/jtd.2017.06.116 tumors of the lung, pleura, thymus and heart. Lyon: IARC PRESS, 2004:152-3.

26. Regnard JF, Magdeleinat P, Dromer C, et al. Prognostic factors and long term results after thymoma resection: a series of 307 patients. J Thorac Cardiovasc Surg 1996;112:376-84.

27. Mangi AA, Wright CD, Allan JS, et al. Adjuvant radiation therapy for stage II thymoma. Ann Thorac Surg 2002;74:1033-37.

28. Chen YD, Feng QF, Lu HZ, et al. Role of adjuvant radiotherapy for stage II thymoma after complete tumor resection Int J Radiat Oncol Biol Phys 2010;78:1400-6.

29. Chang JH, Kim HJ, Wu HG, et al. Postoperative radiotherapy for completely resected stage II or III thymoma. J Thorac Oncol 2011;6:1282-6.

30. Litvak AM, Woo K, Hayes S, et al. Clinical characteristic and outcomes for patients with thymic carcinoma - evaluation of Masaoka staging. J Thorac Oncol 2014;9:1810-5.

31. Detterbeck FC. Evaluation and treatment of stage I and II thymoma. J Thorac Oncol 2010;5:S318-22.

32. Filosso PL, Galassi C, Ruffini E, et al. Thymoma and the increased risk of developing extrathymic malignancies: a multicentre study. Eur J Cardiothorac Surg 2013;44:21924; discussion 224.

33. Okuma Y, Hosomi Y, Takagi Y, et al. Clinical outcomes with chemotherapy for advanced thymic carcinoma. Lung cancer 2013;80:75-80.

34. Girard N, Ruffini E, Marx A, et al. Thymic epithelial tumors: ESMO clinical practice guidelines for diagnosis, treatment and follow up. Ann Oncol 2015;26:v40-55. 